\title{
Online Non-rigid Structure-from-motion based on a keyframe representation of history
}

\author{
Simon Donné, Ljubomir Jovanov, Bart Goossens, Wilfried Philips, Aleksandra Pižurica \\ Department of Telecommunications and Information Processing (TELIN) \\ Ghent University \\ Ghent, Belgium \\ \{Simon.Donne, Ljubomir.Jovanov, Bart.Goossens, Wilfried.Philips, \\ Aleksandra.Pizurica\}etelin.ugent.be
}

\section{ABSTRACT}

Non-rigid structure-from-motion in an on-line setting holds many promises for useful applications, and off-line reconstruction techniques are already very advanced. Literature has only recently started focusing on on-line reconstruction, with only a handful of existing techniques available. Here we propose a novel method of history representation which utilizes the advances in off-line reconstruction. We represent the history as a set of keyframes, a representative subset of all past frames. This history representation is used as side-information in the estimation of individual frames. We expand the history as previously unseen frames arrive and compress it again when its size grows too large. We evaluate the proposed method on some test sequences, focusing on a human face in a conversation. While on-line algorithms can never perform as well as off-line methods as they have less information available, our method compares favourably to the state of the art off-line methods.

\section{INTRODUCTION}

One of the most important problems in computer vision today is the reconstruction of the $3 \mathrm{D}$ geometry of a scene based on one or more cameras capturing 2D image sequences. Non-rigid structure-from-motion is one of the fundamental computer vision problems, with a large number of potential applications such as:

- 3D video: conversion of 2D movies into 3D,

- Human-computer interface (HCI): pose and gesture estimation of the user,

- Minimal-invasion surgery: extraction of 3D information from laparoscopic images to provide surgeons with more detailed information.

These are only some important applications of these techniques, illustrating the importance of accurate and on-line 3D reconstruction. In this paper we focus on a teleconference scenario: we wish to create a more immersive user experience by only using a single $2 \mathrm{D}$ camera. The goal is to estimate the $3 \mathrm{D}$ coordinates for a series of feature points from an input stream of their corresponding 2D observations, and to do so in an on-line fashion. 
The input 2D coordinates are assumed to be reasonably accurate, but our model includes input noise, for example from feature tracker inaccuracies. This paper focuses on the case of only one camera. We make no further assumptions about this camera: it may be either static or moving compared to the object and we do not restrict ourselves to a given camera model in this stage, either perspective or orthographic.

We say that the (external) feature tracker detects $J$ feature points on the surface and that the camera is represented by $C$ parameters. Accordingly, in each frame we wish to estimate $3 J 3 \mathrm{D}$ coordinates and $C$ camera parameters using $2 J$ observed $2 \mathrm{D}$ coordinates: the problem is ill-posed without any restrictions on the possible solutions. The two major challenges are finding the best solution to this ill-posed problem and doing so in an on-line fashion. While the existing literature offers a plethora of solutions to the ill-posed problem in an off-line scenario, on-line non-rigid structure-from-motion has only recently been receiving attention with just a handful of publications at the time of writing [1-3].

\section{EXISTING METHODS}

Non-rigid structure-from-motion is in itself an ill-posed problem: at each input frame we wish to estimate $3 J+C$ unknowns based on only $2 J$ input values, where the camera parameters may or may not include movement depending on the specifics of the method used. To lower the amount of unknowns that need to be estimated, we assume a certain degree of knowledge about the scene. The various methods are generally classified by the type of knowledge they assume.

\subsection{Template-based Methods}

The first option is to assume that the object being reconstructed is well-known and that all of its possible behaviours are collected into a so-called template. As illustrated in the literature, a linear space can adequately represent deformations of a real, physical object such as a human face [4-6]. Such a template effectively restricts the possible 3D point clouds of the object to a $K$-dimensional subspace of the $3 J$-dimensional space, with the template containing the known subspace basis. This means we must estimate $K+C$ unknowns based on $2 J$ input values, which is more likely to be well-determined. Naturally, the dimensionality of the subspace is assumed to be significantly lower than $3 J$. If this were not the case, there is little advantage from a template-based method.

\subsection{Shape Basis Methods}

Unfortunately, templates are often missing due to the lack of any accurate knowledge about the deforming object. We must therefore make less stringent assumptions to reduce the number of unknowns. A relaxation of the assumption of the template methods is that the possible constellations of the object's point cloud are indeed restricted to a $K$-dimensional subspace, but that the basis of this subspace is unknown and must be estimated as well. 


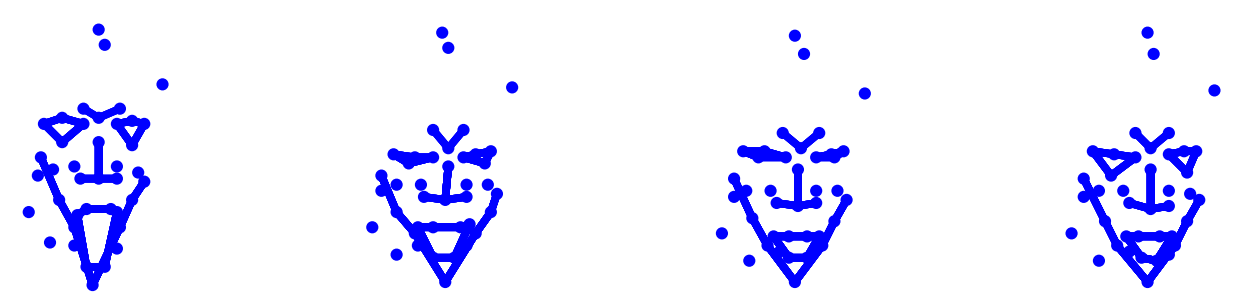

Fig. 1: Illustration of a shape basis extracted with a shape space method.

In this case $K+C$ unknowns have to be estimated for each frame, as well as $3 J K$ unknowns globally for the subspace basis [7]. Figure 1 shows an example of an estimated shape basis, and we can see that this is similar to what one would expect a template to contain.

In the seminal work of [7] the shape basis and the coefficients are extracted through matrix factorization. Several methods improve this seminal method, either in the method of estimation or in the modelling of the object. Some of the most important approaches consist of:

- an extension to non-linear manifolds for the possible constellations [8-11];

- the use of a perspective rather than an orthographic camera model $[12,13]$;

- the use of Bayesian estimation [14, 15];

- the handling of missing data points [16].

One of these approaches, probabilistic principal component analysis (PPCA) [14] is one of the best performing methods when in the input is perturbed by noise, courtesy of its explicit noise modelling. It is the one we choose to champion for the shape basis methods in later comparisons. Moreover, this method is computationally faster than most other methods, which is another point of importance considering our goal of an on-line reconstruction. This method is explained in more detail later in this section, because our proposed method relies on the same modelling and on the same approach for the actual reconstruction.

To the best of our knowledge there exist, at of the time of writing, only a handful of on-line non-rigid structure-from-motion methods in the literature. In [1], the authors perform off-line reconstruction on a bootstrap sequence, which results in an initial estimate for the shape basis. Afterwards this basis is used to estimate the subsequent frames sequentially. The shape basis is then expanded using principal component analysis (PCA). While the shape basis representation from [1] tends to collect a large amount of noise over time in noise-perturbed sequences as it will keep adding bases based on noises, it works well in the noise-free case. The authors of [3] utilize an adaptation of PCA more suited for sequential use: Incremental PCA (IPCA). They use a frame window to step through the input sequence and update the shape basis using IPCA. Their method requires a training input set to estimate the prior distribution of shape basis weights, however. Lastly, the use of an adapted Finite Elements Method (FEM) is proposed [2]. The major drawback of this FEM method is that it requires an initialisation step wherein the object behaves rigidly. This is a valid assumption in a large number of cases, but we will focus on scenarios where this requirement is not necessarily fulfilled. 


\subsection{Trajectory Basis Methods}

Whereas shape basis approaches attempt to model spatial coherence, trajectory basis methods exploit temporal coherence: there is a high correlation between subsequent locations of a given point. These methods model the point trajectories as elements of a $K$-dimensional subspace. The optimal basis for each input sequence can be estimated through principal component analysis (PCA), but research has shown this basis largely coincides with that of the discrete cosine transform (DCT) [17, 18]. A related method models the camera as smoothly moving rather than the points: Column Space Fitting [19], which we classify under trajectory basis methods in this overview. It first estimates the camera behaviour and then uses this knowledge to perform reconstruction more effectively. It is chosen to champion for the trajectory basis methods in later comparisons.

\subsection{Details of PPCA}

Because our method uses the estimation framework from [14] we repeat the basics here. This approach is based on the Bayesian modelling of the tracking errors and the input noise. Let us $\mathbf{s}_{j, t}$ as the $3 \mathrm{D}$ coordinates of point $j, \mathbf{d}_{t}$ as the $3 \mathrm{D}$ camera translation, $\mathrm{R}_{t}$ as the camera matrix and $c_{t}$ as the camera scaling factor,(all in the $t^{t h}$ frame). Under the assumption of Gaussian measurement noise $\mathbf{n}_{j, t} \sim \mathcal{N}\left(0 ; \sigma^{2}\right.$ I $)$ we can then express the $2 \mathrm{D}$ observation $\mathbf{p}_{j, t}$ of the $j^{\text {th }}$ point as a weak-perspective projection:

$$
\underbrace{\mathbf{p}_{j, t}}_{2 \times 1}=\underbrace{c_{t} \mathrm{R}_{t}}_{2 \times 3}(\underbrace{\mathbf{s}_{j, t}}_{3 \times 1}+\underbrace{\mathbf{d}_{t}}_{3 \times 1})+\underbrace{\mathbf{n}_{j, t}}_{2 \times 1}
$$

In matrix notation, the observed locations of all points in a given frame $t$ are concatenated vertically into $\mathbf{p}_{t}$ :

$$
\underbrace{\mathbf{p}_{t}}_{2 J \times 1}=\underbrace{\mathrm{G}_{t}}_{2 J \times 3 J}(\underbrace{\mathbf{s}_{t}}_{3 J \times 1}+\underbrace{\mathbf{D}_{t}}_{3 J \times 1})+\underbrace{\mathbf{n}_{t}}_{2 J \times 1}
$$

In this equation, $\mathrm{G}_{t}$ contains $J$ copies of $c_{t} \mathrm{R}_{t}$ on its diagonal, and other entities are the vertically concatenated versions of their counterparts in Equation 1 . We now assume $\mathbf{s}_{t}$ to be an element of a $K$-dimensional manifold:

$$
\underbrace{\mathbf{s}_{t}}_{3 J \times 1}=\underbrace{\overline{\mathbf{s}}}_{3 J \times 1}+\underbrace{\mathrm{V}}_{3 J \times K} \underbrace{\mathbf{z}_{t}}_{K \times 1},
$$

where $\overline{\mathbf{s}}$ is an average $3 \mathrm{D}$ shape, and V holds the shape basis vectors in its columns, which are weighted with the deformation coefficients contained in $\mathbf{z}_{t}$.

Subsequently, the authors of [14] place a Gaussian prior on the deformation weights: $\mathbf{z}_{t} \sim \mathcal{N}(0 ; \mathrm{I})$. The estimation of $\mathrm{R}_{t}, \overline{\mathbf{s}}, \mathrm{V}$ and $\mathbf{z}_{t}$ then amounts to a Bayesian scheme, which is called probabilistic principal component analysis (PPCA). Specifically, an expectation-maximization (EM) optimization is used for estimating the various parameters. One final remark must be given about the localisation: without any fixed reference system, the location of the camera and the object are only estimated up to an affine transform. For ease of use we will assume that the center of the observed object is the origin (and that this center does not fluctuate markedly due to deformations). We will then express any movement of either the real-life camera or the real-life object as movements of our virtual camera, fixing the object's center at the virtual origin. 


\section{THE PROPOSED ON-LINE RECONSTRUCTION}

We can identify two large elements in any on-line method: a method for history representation and one for the sequential processing of the input. In an initial attempt for on-line reconstruction method we performed the EM update equations using the information from a sliding temporal window for all of the unknowns. Due to small frame windows and slow-moving cameras, this strategy typically resulted in a degeneration of the shape basis and the resulting reconstruction because the frame window did not contain enough vantage points for the object. In this case, the latest estimations of the various unknowns were used as a history representation: obviously this history representation was too simplistic. This section comprises the selection of a representative subset, both on artificial 2D point clouds and our specific problem, and the overview of our proposed method.

\subsection{History Representation with keyframes}

A key element of any sequential or on-line algorithm is the need to remember past input. Clearly, it is intractable to simply memorize all past frames of a theoretically infinite sequence. We propose to use a set of keyframes as a representation of all past input frames; a sparse sampling of history, so to speak. We extract the shape basis for the shape subspace required for the reconstruction from this history representation, but we retain the entire subset as the history representation. The challenge is now the selection of such a set of representative frames from all of the input frames.

General Subset Selection The main goal of the keyframe selection procedure is to select a set of frames which represent all the possible deformations of the object from as many vantage points as possible. In order to achieve this goal, the selection procedure should exclude all of the frames that do not contribute any additional information to the shape basis, eliminating duplicate frames from the keyframe set.

A subset of representative frames is selected from the full set, having much fewer elements. Due to the combinatorial explosion, the number of possible ways to select a subset from a larger set grows very quickly with the size of the full set. Therefore, an exhaustive comparison of all possible subsets of keyframes is implausible and we resort to a heuristic subset selection. Assuming that we can represent the elements of the set as points in a Euclidean space, our goal is to select a subset, the elements of which are uniformly distributed throughout the bounds of the full set.

To select a subset with a given metric, we use backward elimination. By starting from the full set and removing one element at a time according to a local criterion we remove the element whose exclusion from the subset results in the best change of the metric, e.g. one of the two elements lying closest together when maximizing the minimum distance: the one lying closest to the rest of the subset.

We illustrate four different metrics to maximize: the minimum distance between any two elements of the subset, the mean distance between all elements of the subset, the mean distance between the elements of the subset and those of the full set, and the differential entropy of the subset. These metrics are heuristically chosen so that their maximization results either in a uniform density over the bounds (the minimum distance and the entropy), or in an accurate representation of the bounds (the mean distance). 

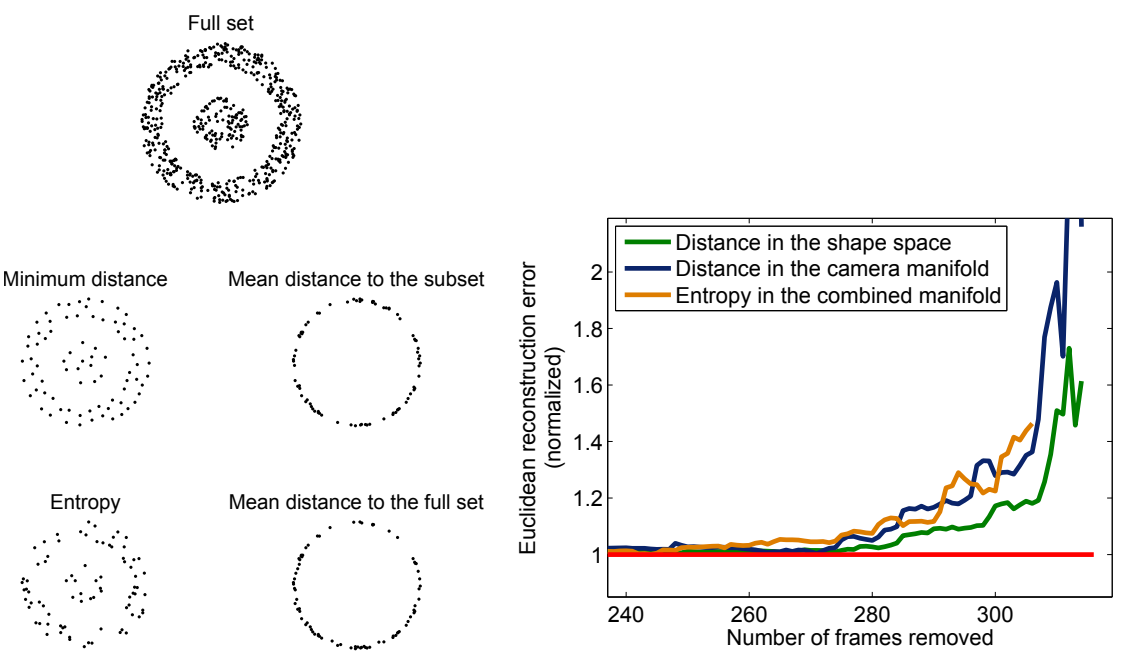

Fig. 2: Demonstration of the subset Fig. 3: Keyframe selection using several metrics. selection metrics on an artificial 2D The input sequence is a 316-frame sequence of a point cloud. person talking from [14]

The mean distance to the full set is included because it is computationally faster than the mean distance to the subset. For the first three metrics we require a meaningful distance to be defined between any two elements in the set, a drawback the differential entropy does not suffer from. To estimate the differential entropy, we use a Matlab wrapper for the TIM, an open-source C++ library for efficient estimation of informationtheoretic measures [20].

The result of each of these strategies on the selection of a representative subset for an artificial 2D point cloud are shown in Figure 2. We can see that maximizing the minimum distance between any two points in the subset yields the most representative cloud, while maximizing the mean distance represents the edges well. The entropy metric gives disappointing results, which we assume largely to be the result of the difficulty of estimating the entropy of a continuous variable based on a small number of samples.

Subset selection for keyframe selection Results shown in Figure 2 demeonstrate that it is possible to select a representative subset of $2 \mathrm{D}$ points from a much larger set. In this section we investigate whether this result also holds for selecting a set of keyframes from the full set of frames, as illustrated in Figure 4. The set of keyframes is said to be representative to the full set if the reconstruction error is not significantly affected by restricting the estimation of the shape basis to the set of keyframes rather than the full set. To this end, three metrics for the selection of keyframes are investigated:

- the minimum distance in the shape space,

- the minimum distance in the camera manifold,

- the entropy in the combined shape-camera space. 


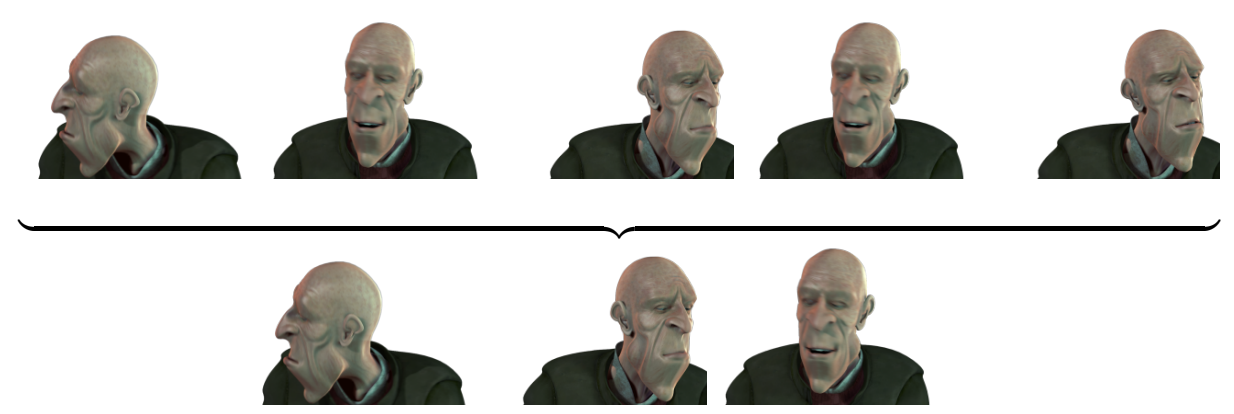

Fig. 4: Illustration of how the keyframe selection should work: any duplicate or near identical frames should be eliminated. Images courtesy of the open-source video Elephant's Dream [21], featuring the character Proog.

We use the Euclidian distance between deformation coefficients as the distance metric in the shape space. In the camera manifold, the distance between two camera parameter vectors is defined as the euclidean distance between a given unit vector transformed to the cameras' reference system. The differential entropy is retained as a metric in this paper because it allows us to combine the deformation coefficients and the camera parameters into a single metric, which is not straightforward using distance-based metrics and would require extensive research.

Figure 3 shows that the minimum distance on the shape manifold results in a lower reconstruction error than the other methods, indicating that the observation of the different deformations is more important to the overall accuracy than the observation from different vantage points. The results were obtained on a 316-frame sequence from [14] consisting of a person talking to the camera. At each step, we eliminate the frame which maximizes the respective metric and perform PPCA reconstruction restricting the estimation of the shape basis to the keyframes. Figure 3 also shows that for keyframe set sizes of about 35 frames there is little loss in accuracy.

\subsection{Overview of the Proposed Algorithm}

We start by performing a 3D reconstruction of a bootstrap sequence with an existing, offline, reconstruction method. For this, any off-line reconstruction algorithm can be used, but we have chosen the PPCA method [14] for simplicity. The length of the bootstrap sequence must be chosen with some care: choosing it too short will result in a rough initialization, while choosing it too long will increase the initialisation time. For the sequences used in this paper we have found a bootstrap length of 60 frames to be a good middle ground. We select a subset of frames from the bootstrap sequence which accurately represents the whole of the bootstrap sequence, through the already discussed keyframe selection. A rough initial reconstruction can be performed for the bootstrap window (through a limitation of the iteration count), which is sufficient for keyframe selection and which we improve only for the selected keyframes.

Throughout the execution of the program we will add frames to the keyframe set, and we cull the keyframe set using the subset selection whenever its cardinality exceeds 


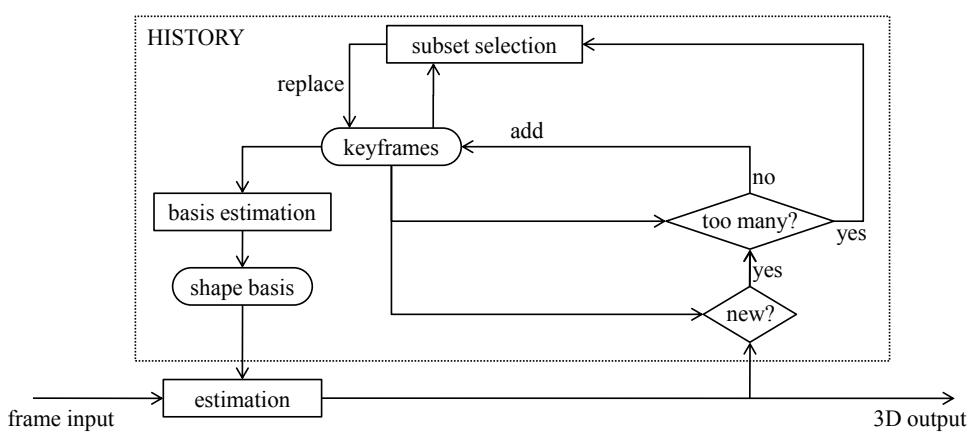

Fig. 5: Overview of our proposed method.

a certain imposed size (heuristically chosen to be 1.5 times the initial size). The initial history size is also chosen manually, and tests have shown (see for example Figure 3) that a set of 30 frames is an acceptable choice.

For the on-line processing, we reconstruct each frame sequentially using the shape basis we have extracted from the keyframe set, initialising the camera matrix using the last estimated value. This consists of alternately optimizing the camera parameters and the deformation coefficients for a set number of iterations using the update steps from [14]. In the next step we add the newly processed frame to the keyframe set if it represents a deformation or vantage point which is not yet represented in said set, i.e. if the subset selection metric does not lower significantly when including the newly processed frame. At the point where the keyframe set has changed significantly, i.e. it has grown to a predefined threshold, we select a new subset of the large keyframe set to serve as the new history representation from now on. Finally, we extract an updated shape basis from the updated history and continue with the on-line processing. The update of the keyframe set and the extraction of the new shape basis can be done in parallel with the on-line reconstruction using parallel programming paradigms (OpenMP or GPGPU). An overview of the proposed algorithm is given in the form of a flowchart in Figure 5.

\subsection{GOP Processing}

Estimating the 3D shape one frame at a time incurs a large amount of overhead, because we are performing calculations on small matrices: the overhead of the calculations is large compared to the complexity of the calculations. Group-of-pictures (GOP) processing is possible in the proposed method due to the nature of the optimization equations, which results in a lower average overhead per frame. Processing multiple frames at the same time comes with the disadvantage of a higher latency, and therefore the end-user will have to decide which point of the trade-off is optimal for their particular application.

To illustrate the relationship between GOP size, processing speed and latency, we vary the GOP size from 1 to 10 and scatter the points $\left(F P S_{x}\right.$, Latency $\left._{x}\right)$ in our Matlab implementation, as visible in Figure 6. The latency displayed in the graph is the minimum latency: to compute it we ignore the fact that the first frame of a GOP must wait until the last frame of its GOP is observed until the estimation of the GOP can begin. Therefore, 


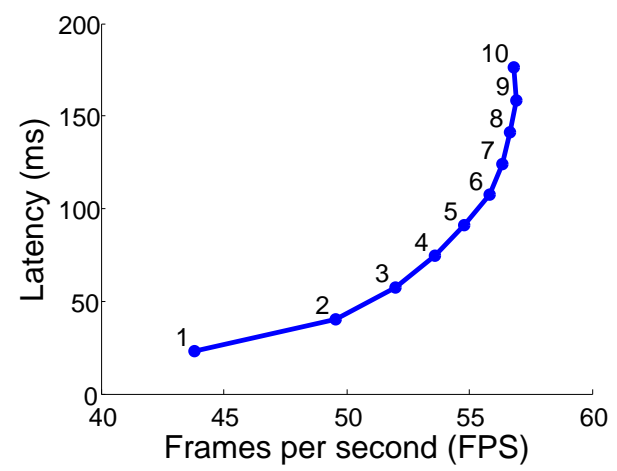

Fig. 6: Relationship between GOP size, pro- Fig. 7: Causal comparison between two excessing speed and minimum latency. Each isting methods and our proposed method. data point is labeled by the GOP size re- The input sequence is consists of a person sponsible for it.

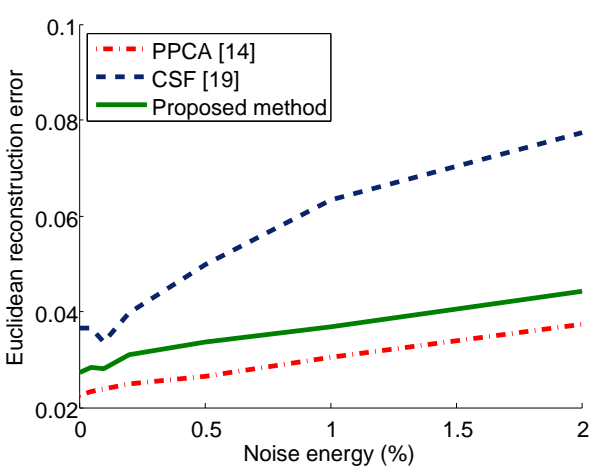

talking, courtesy of [14].

the displayed latency is the latency for the last frame of a GOP. The actual choice of the GOP size depends on the application: a large GOP size may be applicable to off-line reconstruction of a very long sequence because current off-line reconstruction methods typically scale badly with rising sequence length, in computational complexity and/or required memory. In this case, latency is of little interest and the goal is to maximize the throughput while avoiding the high memory use and complexity of off-line methods.

\section{RESULTS}

\subsection{Quantitative Results}

In this section we present the result of the proposed approach and compare it to some state-of-the-art methods. It is important to note that the proposed method is restricted to causal processing of the input sequence: we reconstruct frame $t$ using only the observations from frames 1 through $t$. The existing methods work off-line on the whole video sequence and are not restricted by causality: for a more fair comparison we can restrict these to causal reconstruction as well. We do this because we want to focus our evaluation on the effectiveness of our history representation. Assuming the methods are allocated a bootstrap window of $B$ frames, we can restrict them to causal workings by reconstructing frame $t$ using only the frames $1, \ldots, t$. Clearly, this is not a practical way to perform reconstruction, but it gives an indication of the performance of other methods in a causal setting. Figure 7 shows the comparison between the proposed method and some state-of-the-art methods [19, 14]. While the proposed method can never perform a reconstruction of the same accuracy as its off-line variant, because it does not retain the same amount of information on previous frames, our proposed method still compares favourably to the off-line PPCA method and outperforms other state of the art methods. Our proposed method has also inherited the noise robustness of the Bayesian modelling from the off-line PPCA, as illustrated by the equality of the slopes. 
These graphs of course do not reflect the immense advantage of the on-line aspect of our method. There is no entry for the first online method by [1] because it uses a threshold for the error as an indicator of whether or not the model should be expanded. The resulting line in the graph is therefore constant and does not reflect the downsides of the method (extraordinary computational complexity with rising noise). The graph was produced by perturbing the 316-frame sequence from [14] with zero-mean Gaussian noise (AWGN), reconstructing the perturbed sequence with the various methods and afterwards computing the average 3D error between the reconstructed point cloud and the ground truth. The specific parameter values for our method were: a 60 -frame bootstrap length, a 40-frame history representation size and 25 EM iterations per frame.

\subsection{Qualitative Results}

Figure 8 puts these reconstruction errors into perspective: all three methods manage an accurate reconstruction of the face from the sequence from [14]. We also present a reconstruction by the three methods of the sequence extracted from Elephant's Dream [21] in Figure 9. For this comparison, we extract the projected points from the video's source and pass them as input to the reconstruction methods. Because the full mesh of the Proog's face has over 4000 points, we manually selected a subset of 197 points to perform reconstruction on. After reconstruction, the resulting 3D mesh is visualized by using the original texture from the movie sources.

\section{CONCLUSION}

In this paper we have proposed a new method for on-line non-rigid structure-from-motion based on keyframe selection. While the literature on off-line 3D reconstruction has received a lot of attention and several accurate techniques exist, relatively few ventures have been made concerning on-line operation. A new method of history representation using a set of keyframes is described and evaluated, comparing favourably to existing methods, performing similarly to its off-line variant and outperforming other off-line state-of-the-art methods.

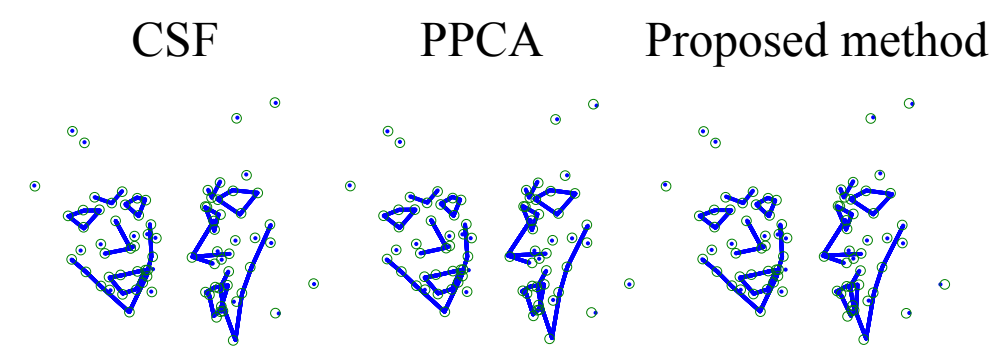

Fig. 8: Visual comparison between three methods. The top row shows the original view point, and the bottom row shows an alternate view point. Lines and points show the projection of reconstructed 3D points and their connections, while small circles are centered around the ground truth locations. 


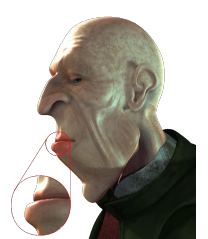

(a) movie

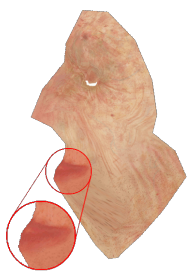

(b) CSF

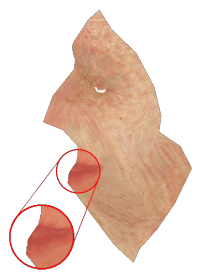

(c) PPCA

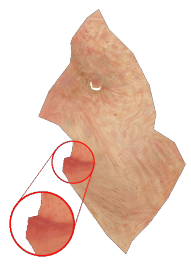

(d) Proposed method

Fig. 9: Visual comparison between two existing methods and our proposed method on a sequence extracted from Elephant's Dream [21].

We see two clear options for future work on on-line non-rigid structure-from-motion: improving the history representation and improving the estimation of the separate frames. Sequential statistical estimation, which is used to great effect in other fields, may offer a more theoretical approach to history representation. On the other hand, the estimation of the separate frames can no doubt benefit from existing methods in off-line reconstruction. The exploitation of the temporal coherence through implementation of a linear dynamic system as in [14], the PPCA-specific improvement of the camera update equation as in [22], or other techniques from off-line state-of-the-art offer possibilities for improvement.

\section{ACKNOWLEDGEMENTS}

The research for this paper was started in a master's thesis at Ghent University (at the TELIN department) and finished during the start of a PhD funded by the BOF under grant number 01D21213. Special thanks go to Erwin Six and Donny Tytgat at the Alcatel Bell Labs in Antwerp for their help and insights during the early stages of the research.

\section{References}

1. Marco Paladini, Adrien Bartoli, and Lourdes Agapito. Sequential non-rigid structure-frommotion with the 3d-implicit low-rank shape model. In Proceedings of the 11th European conference on Computer vision: Part II, ECCV'10, pages 15-28, Berlin and Heidelberg, 2010. Springer-Verlag.

2. Antonio Agudo, Begoa Calvo, and J.M.M. Montiel. 3d reconstruction of non-rigid surfaces in real-time using wedge elements. In Computer Vision ECCV 2012. Workshops and Demonstrations, volume 7583 of Lecture Notes in Computer Science, pages 113-122. 2012.

3. Lili Tao, Stephen J. Mein, Wei Quan, and Bogdan J. Matuszewski. Recursive non-rigid structure from motion with online learned shape prior. Computer Vision and Image Understanding, 117(10):1287 - 1298, 2013.

4. Volker Blanz and Thomas Vetter. A morphable model for the synthesis of $3 \mathrm{~d}$ faces. In Proceedings of the 26th annual conference on Computer graphics and interactive techniques, SIGGRAPH '99, pages 187-194, New York and NY and USA, 1999. ACM Press/AddisonWesley Publishing Co. 
5. Shu-Fan Wang and Shang-Hong Lai. Estimating $3 \mathrm{~d}$ face model and facial deformation from a single image based on expression manifold optimization. In Proceedings of the 10th European Conference on Computer Vision: Part I, ECCV '08, pages 589-602, Berlin and Heidelberg, 2008. Springer-Verlag.

6. P. Paysan, R. Knothe, B. Amberg, S. Romdhani, and T. Vetter. A 3d face model for pose and illumination invariant face recognition. In Advanced Video and Signal Based Surveillance and 2009. AVSS '09. Sixth IEEE International Conference on, pages 296 -301, sept. 2009.

7. C. Bregler, A. Hertzmann, and H. Biermann. Recovering non-rigid $3 d$ shape from image streams. In Computer Vision and Pattern Recognition and 2000. Proceedings. IEEE Conference on, volume 2, pages 690-696 vol.2, 2000.

8. V. Rabaud and S. Belongie. Re-thinking non-rigid structure from motion. In Computer Vision and Pattern Recognition and 2008. CVPR 2008. IEEE Conference on, pages 1 -8, june 2008.

9. A. Shaji and S. Chandran. Riemannian manifold optimisation for non-rigid structure from motion. In Computer Vision and Pattern Recognition Workshops and 2008. CVPRW '08. IEEE Computer Society Conference on, pages 1 -6, june 2008.

10. Joo Fayad, Alessio Del Bue, Lourdes de Agapito, and Pedro Aguiar. Non-rigid structure from motion using quadratic deformation models. In $B M V C$. British Machine Vision Association, 2009.

11. P.F.U. Gotardo and A.M. Martinez. Kernel non-rigid structure from motion. In Computer Vision (ICCV) and 2011 IEEE International Conference on, pages 802 -809, nov. 2011.

12. Jing Xiao and Takeo Kanade. Uncalibrated perspective reconstruction of deformable structures. In Proceedings of the Tenth IEEE International Conference on Computer Vision - Volume 2, ICCV '05, pages 1075-1082, Washington and DC and USA, 2005. IEEE Computer Society.

13. Richard Hartley and Ren Vidal. Perspective nonrigid shape and motion recovery. In In Proc. European Conference on Computer Vision, pages 276-289, 2008.

14. Lorenzo Torresani, Aaron Hertzmann, and Christoph Bregler. Non-rigid structure-frommotion: Estimating shape and motion with hierarchical priors. IEEE Transactions on Pattern Analysis and Machine Intelligence, 2008.

15. Huiyu Zhou, Xuelong Li, and A.H. Sadka. Nonrigid structure-from-motion from 2-d images using markov chain monte carlo. Multimedia and IEEE Transactions on, 14(1):168 -177, feb. 2012.

16. Sung Joo Lee, Kang Ryoung Park, and Jaihie Kim. A sfm-based 3d face reconstruction method robust to self-occlusion by using a shape conversion matrix. Pattern Recogn., 44(7):14701486, July 2011.

17. Ijaz Akhter, Yaser Sheikh, Sohaib Khan, and Takeo Kanade. Trajectory space: A dual representation for nonrigid structure from motion. IEEE Transactions on Pattern Analysis and Machine Intelligence (TPAMI), 2011.

18. Ijaz Akhter, Yaser Ajmal Sheikh, Sohaib Khan, and Takeo Kanade. Nonrigid structure from motion in trajectory space. In Neural Information Processing Systems, December 2008.

19. Paulo F.U. Gotardo and Aleix M. Martinez. Computing smooth time trajectories for camera and deformable shape in structure from motion with occlusion. IEEE Transactions on Pattern Analysis and Machine Intelligence, 33:2051-2065, 2011.

20. Kalle Rutanen. Tim, information-theoretic measures in matlab. http://www.cs.tut.fi/ timhome/tim/tim.htm.

21. Orange Open Movie Team. Elephant's dream. http://www.elephantsdream.org/.

22. Chengchao Qu, Hua Gao, and Hazım Kemal Ekenel. Rotation update on manifold for non-rigid structure from motion. IEEE International Conference on Image Processing, oct. 2012. 